EUROPEAN ORGANIZATION FOR NUCLEAR RESEARCH

CERN-PS DIVISION

CERN/PS 99-049 (CA)

\title{
COLLECTIVE EFFECTS IN THE CERN-PS BEAM FOR LHC
}

\author{
R. Cappi, R. Garoby, E. Métral
}

Abstract

This paper is an updated review of the collective effects observed and predicted in The CERN-PS machine for the LHC beam.

Workshop on Instabilities of High Intensity Hadron Beams in Rings At Brookhaven National Laboratory, Upton, N.Y., June 28 to July 1, 1999

Geneva, Switzerland

2 September 1999 


\title{
Collective effects in the CERN-PS beam for LHC
}

\author{
R. Cappi, R. Garoby, E. Métral
}

CERN, PS Division, 1211 Geneva 23, Switzerland

\begin{abstract}
This paper is an updated review of the collective effects observed and predicted in the CERN-PS machine for the LHC beam.
\end{abstract}

\section{INTRODUCTION}

The PS machine as part of the LHC injector chain has to provide to the SPS a proton beam with specific characteristics [1]. To summarize, in the longitudinal plane the main problem is to generate a train of very short bunches $(\sim 3.8 \mathrm{~ns})$ spaced by $25 \mathrm{~ns}$, starting from very long bunches ( $200 \mathrm{~ns})$ coming from the PSB. While, in the transverse domain, the main issue is to provide a beam of high brightness (i.e. intensity to emittance ratio), with an intensity of $1.4 \times 10^{13} \mathrm{p} / \mathrm{p}$ (for the ultimate beam) and normalized rms transverse emittances of $3 \mu \mathrm{m}$.

The solution adopted is to accelerate in the PSB a beam with the right transverse emittance, but half the intensity, and inject two pulses into the PS machine at $1.4 \mathrm{GeV}$ kinetic energy. The total circumference of the four PSB rings being equal to the PS circumference, a necessary condition, in order to fill only one half of the PS with a single PSB shot, is to use a $h=1 \mathrm{RF}$ system in the PSB. The second half of the PS is filled with a second shot, $1.2 \mathrm{~s}$ later ( $4+4=8$ bunches). The 8 bunches are captured by the PS RF system on $h=8$ and then split into 16 with an adiabatic change of harmonic number from 8 to 16 . They are subsequently accelerated to $26 \mathrm{GeV} / \mathrm{c}$ where the beam is debunched and rebunched at $40 \mathrm{MHz}$ to provide the $25 \mathrm{~ns}$ spacing. Finally, the 84 bunches are compressed to $3.8 \mathrm{~ns}$ with a $2^{\text {nd }}$ harmonic RF system $(80 \mathrm{MHz}$ cavities, $300 \mathrm{kV}$ each) and fast extracted to the SPS. This paper reports experiments performed to study and cure both longitudinal and transverse instabilities.

\section{LONGITUDINAL INSTABILITIES}

\section{Coupled-Bunch Instabilities}

To provide the required LHC beam characteristics many modifications have been recently made, in particular in the RF system [2]. New low-level beam controls to

accelerate the beam on $h=8$ and $h=16$ (instead of the previous $h=20$ ) have been implemented and extra cavities have been installed in the ring (two $40 \mathrm{MHz}$ and three 
$80 \mathrm{MHz}$ ). Accelerating the beam on $h=8$, the frequency spectrum of the beam and the tuning frequency of the ferrite cavities have changed. A new coupled-bunch instability has appeared in the vicinity of the $10^{\text {th }}$ harmonic of the revolution frequency (i.e. coupled-bunch mode $n=2$ or 6 ) at about $3.5 \mathrm{GeV} / \mathrm{c}$ (see Fig. 1(a)). A damping system has been implemented by filtering the wall-current monitor signal at the instability frequency and reinjecting it into a pair of ferrite accelerating cavities (see Fig. 1(b)) [3].

(a)

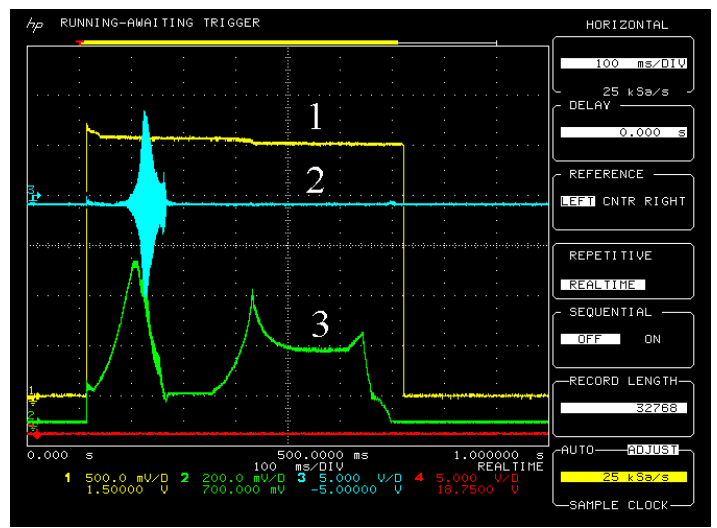

(b)

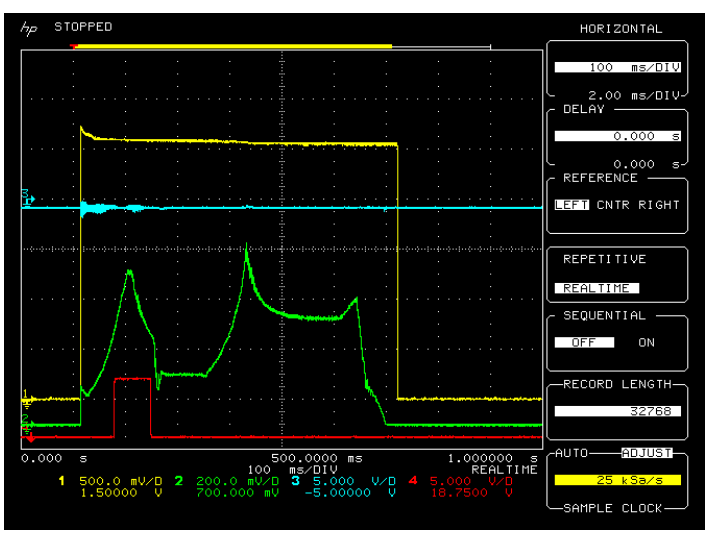

FIGURE 1. (a) Trace 1: Circulating beam current. Trace 2: Wall-current monitor signal filtered at $f=10 f_{0}+f_{\mathrm{s}}$. Trace 3: Detected wall-current monitor (i.e. bunch height). Time scale: $100 \mathrm{~ms} / \mathrm{div}$. (b) Same as Fig. 1(a), but with the longitudinal feedback in operation.

\section{Microwave Instabilities}

For the LHC beam, the 8 bunches injected into the PS, split into 16 at $3.5 \mathrm{GeV} / \mathrm{c}$, have to be "transformed" into a bunch train of 84 bunches (with a bunch spacing of $25 \mathrm{~ns}$ ) before extraction to SPS. This implies a debunching-rebunching of the beam on $h=84(40 \mathrm{MHz})$. Moreover, because of longitudinal and transverse acceptances in the receiving machine their longitudinal emittances should not be larger than $\sim 0.4 \mathrm{eVs} /$ bunch. Unfortunately, microwave instabilities develop at the end of the debunching procedure, increasing the final longitudinal emittance by a factor 1.5 with respect to the desired value (see Figs. 2(a) and 2(b)).

The Keil-Schnell formula predicts a longitudinal wide-band impedance $Z / n$ of $\sim 300 \Omega$, which is incompatible with the $20 \Omega$ measured using other methods. HOMs in the $114 \mathrm{MHz}$ pill-box cavities used for the lepton acceleration are suspected. Some UHF signals have been detected on the wideband mini-antennas inserted into these cavities. The definitive answer will be known after their removal in 2001, when LEP will be stopped. Moreover, a new scheme has been proposed, which does not use

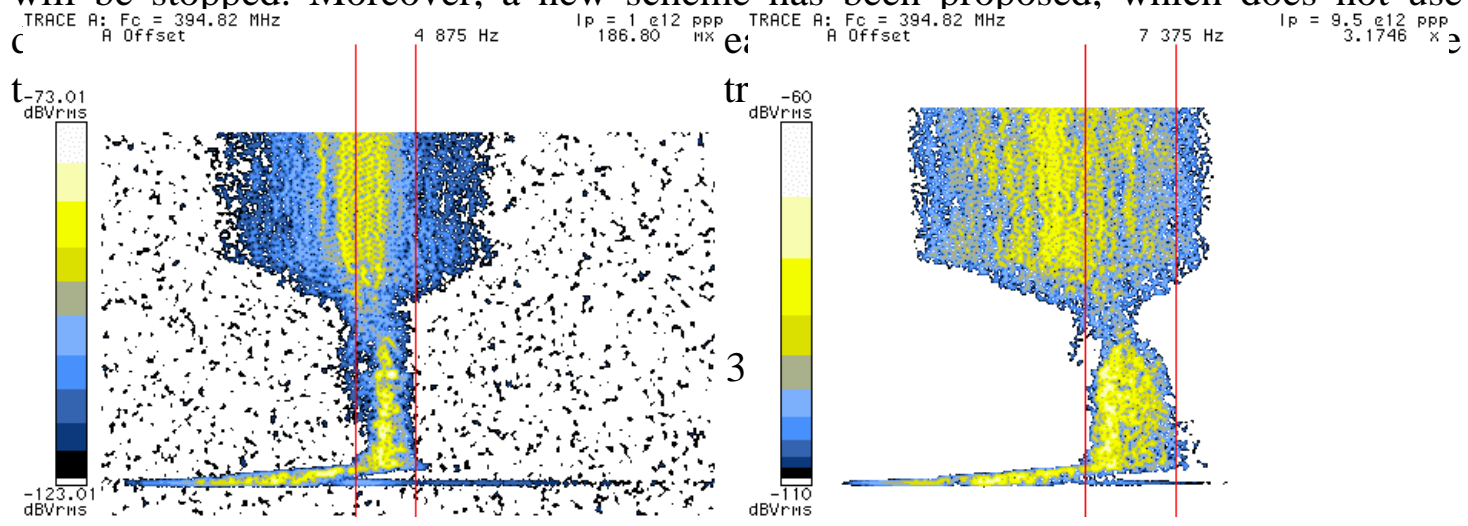


FIGURE 2. (a) Longitudinal Schottky scan spectrogram during the debunching of a low intensity beam $\left(10^{12} \mathrm{p} / \mathrm{p}\right)$. Time goes from top to bottom. Total time window is $\sim 200 \mathrm{~ms}$. In the first $100 \mathrm{~ms}$ the beam is still bunched by the RF voltage, which is adiabatically decreased and then switched OFF. During the following $\sim 50 \mathrm{~ms}$ the beam is debunching with negligible momentum blow-up. The total (relative) momentum spread, indicated by the two line markers, is $0.5 \times 10^{-3}$. The last "transient" is produced by the fast extraction process. (b) Same as Fig. 2(a), but for a higher intensity beam $\left(10^{13} \mathrm{p} / \mathrm{p}\right)$. During the debunching there is a momentum blow-up. The final total (relative) momentum spread is $\sim 0.8 \times 10^{-3}$.

\section{TRANSVERSE INSTABILITIES}

\section{Theory}

\section{Coherent Frequency Shifts of Bunched-Beam Modes}

Sacherer's formula for the transverse coherent frequency shifts of bunched-beam modes is given by [5]

$$
\Delta \omega_{c, m}^{x, y}=(|m|+1)^{-1} \frac{j e \beta I_{b}}{2 m_{0} \gamma Q_{x 0, y 0} \Omega_{0} L} \frac{\sum_{k=-\infty}^{k=+\infty} Z_{x, y}\left(\omega_{k}^{x, y}\right) h_{m}\left(\omega_{k}^{x, y}-\omega_{\xi_{x, y}}\right)}{\sum_{k=-\infty}^{k=+\infty} h_{m}\left(\omega_{k}^{x, y}-\omega_{\xi_{x, y}}\right)}, \text { (1) }
$$

where $\omega_{k}^{x, y}=\left(k+Q_{x 0, y 0}\right) \Omega_{0}+m \omega_{s}$. The transverse bunch spectra of mode $|m|$ are given by

$$
h_{m}\left(\omega-\omega_{\xi_{x, y}}\right)=(|m|+1)^{2} \frac{1+(-1)^{|m|} \cos \left[\left(\omega-\omega_{\xi_{x, y}}\right) \tau_{b}\right]}{\left[\left(\omega \tau_{b} / \pi\right)^{2}-(|m|+1)^{2}\right]^{2}},
$$

and the PS transverse coupling impedances $Z_{x, y}(\omega)$ are approximated by the sum of the resistive wall $\left(Z_{x}^{R W}(\omega) \approx Z_{c}^{R W}(\omega) \times 0.45\right.$ and $Z_{y}^{R W}(\omega) \approx Z_{c}^{R W}(\omega) \times 0.85$ [6]) and broad band impedances

$$
\begin{gathered}
Z_{c}^{R W}(\omega)=[\operatorname{Sgn}(\omega)+j] \frac{R}{b_{w}^{3}} \sqrt{\frac{2 \rho_{w}}{\varepsilon_{0}|\omega|}}, \\
Z_{x, y}^{B B}(\omega)=\frac{\omega_{r}}{\omega} R_{x, y} /\left[1-j Q\left(\frac{\omega_{r}}{\omega}-\frac{\omega}{\omega_{r}}\right)\right] .
\end{gathered}
$$

Making the numerical computations for the single-bunch beam with nominal intensity (see Appendix), the following results, collected in Table 1, are obtained. 
TABLE 1. Transverse instability growth rates and real frequency shifts of the nominal single-bunch beam for modes $m=0$ to 10 .

\begin{tabular}{ccccccc}
\hline Head-tail mode $\boldsymbol{m}$ & $\mathbf{0}$ & $\mathbf{1}$ & $\mathbf{2}$ & $\mathbf{3}$ & $\mathbf{4}$ & $\mathbf{5}$ \\
\hline Horizontal growth rate $\left[\mathrm{s}^{-1}\right]$ & -95.5 & -49.1 & -34.4 & -28.6 & -26.9 & -11.3 \\
Vertical growth rate $\left[\mathrm{s}^{-1}\right]$ & -207.8 & -104.8 & -71.1 & -54.4 & -45.5 & -39.6 \\
Horizontal frequency shift $[\mathrm{rad} / \mathrm{s}]$ & -1727.4 & -865.1 & -579.0 & -437.2 & -359.8 & -306.2 \\
Vertical frequency shift $[\mathrm{rad} / \mathrm{s}]$ & -5050.4 & -2526.2 & -1685.4 & -1265.5 & -1014.5 & -848.1 \\
\hline & & $\mathbf{6}$ & $\mathbf{7}$ & $\mathbf{8}$ & $\mathbf{9}$ & $\mathbf{1 0}$ \\
\cline { 2 - 6 } & & 2.4 & 0.9 & 0.1 & -0.3 & -0.5 \\
& & -39.5 & -32.9 & -8.9 & -0.8 & -3.8 \\
& & -254.3 & -217.7 & -192.4 & -172.1 & -156.1 \\
& & -733.0 & -655.5 & -582.0 & -512.2 & -462.4 \\
\hline
\end{tabular}

The plot of the transverse instability growth rates as functions of the head-tail mode number is represented in Figure 3.

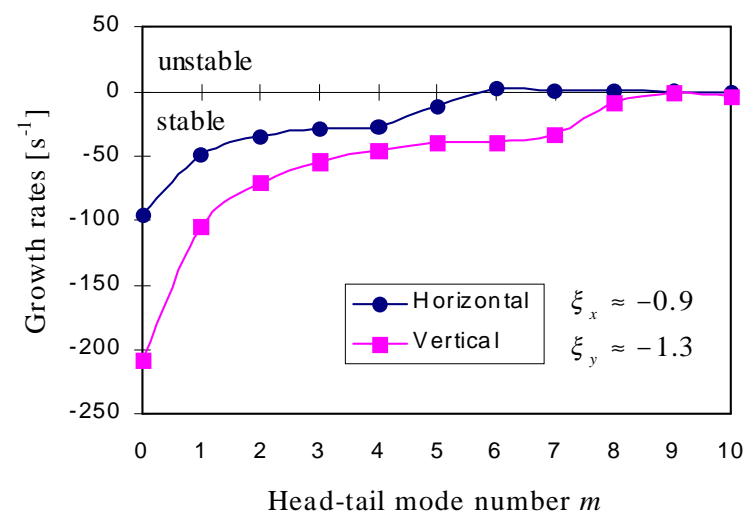

FIGURE 3. Transverse instability growth rates vs. head-tail mode number for the nominal single-bunch beam.

One concludes therefore that the theory, based on the above impedance model, predicts horizontal single-bunch instabilities with most critical head-tail mode number $m=6$.

\section{Stabilization by Landau Damping}

The transverse betatron frequency spreads (half widths at half height) are given analytically by [7]

$$
\Delta \omega_{\mathrm{HWHH}}^{x, y} \approx \frac{3 f_{0} N_{\mathrm{oct}}}{8}\left[\left(\beta_{\mathrm{oct} x, y}^{2} \varepsilon_{x, y}^{\mathrm{rms}}\right)^{2}+\left(2 \beta_{\mathrm{oct} x} \beta_{\mathrm{oct} y} \varepsilon_{y, x}^{\mathrm{rms}}\right)^{2}\right]^{1 / 2} \int_{0}^{l_{\operatorname{mag}}} K_{\mathrm{oct}} d l .
$$

A simplified stability criterion, which is drawn from dispersion relation analysis considering "elliptical" betatron frequency distributions, is given by [8]

$$
\Delta \omega_{\mathrm{HWHH}}^{x, y} \geq \sqrt{3}\left|\Delta \omega_{c, m}^{x, y}\right| .
$$


From the numerical computations, the relations between the transverse betatron frequency spreads and the octupole current, for the nominal single-bunch beam, are given by

$$
\Delta \omega_{\mathrm{HWHH}}^{x}[\mathrm{rad} / \mathrm{s}] \approx 67 I_{\text {oct }}[\mathrm{A}], \quad \Delta \omega_{\mathrm{HWHH}}^{y}[\mathrm{rad} / \mathrm{s}] \approx 85 I_{\text {oct }}[\mathrm{A}] .
$$

Therefore, the theory, based on the above impedance and frequency distribution models, predicts beam stability for $I_{o c t} \approx 6.6 \mathrm{~A}$. Notice that the space-charge component of the impedance has not been taken into account in our calculations (as concerns both instability and damping [7]).

\section{Stabilization by Coupled Landau Damping}

In the presence of linear coupling, but in the absence of external non-linearities, the necessary condition for the stability of the $m$ th mode is that the sum of the transverse instability growth rates, in the absence of both coupling and Landau damping, is negative $[6,9]$

$$
V_{\mathrm{eq} x}^{m}+V_{\mathrm{eq} y}^{m} \leq 0
$$

If Eq. (8) is true, then it is possible to stabilize this mode by increasing the skew gradient and/or by getting closer to the coupling resonance $Q_{h}-Q_{v}=l$. The theoretical stabilizing values of the modulus of the $l$ th Fourier coefficient of the skew gradient are given by

$$
\begin{aligned}
& \left|\underline{\hat{K}}_{0}(l)\right| \geq \\
& \frac{2\left[-Q_{x 0} Q_{y 0} V_{\text {eqx }}^{m} V_{\text {eqy }}^{m}\right]^{1 / 2}}{R^{2} \Omega_{0}} \times \frac{\left[\left(V_{\text {eqx }}^{m}+V_{\text {eqy }}^{m}\right)^{2}+\Omega_{0}^{2}\left(Q_{h}-Q_{v}-l\right)^{2}\right]^{1 / 2}}{-\left(V_{\text {eq } x}^{m}+V_{\text {eqy }}^{m}\right)},
\end{aligned}
$$

where $Q_{h, v}=Q_{x 0, y 0}+U_{\text {eq } x, y}^{m} / \Omega_{0}$ are the horizontal and vertical coherent tunes in the presence of wake fields $\left(U_{\text {eq } x, y}^{m}\right)$, but in the absence of coupling, and

$$
U_{\text {eq } x, y}^{m}=\operatorname{Re}\left(\Delta \omega_{c, m}^{x, y}\right), \quad V_{\text {eq } x, y}^{m}=-\operatorname{Im}\left(\Delta \omega_{c, m}^{x, y}\right),
$$

with $\operatorname{Re}($ ) and $\operatorname{Im}($ ) standing for real and imaginary parts. Furthermore, in the case of coupled-bunch instabilities, the mode numbers are related by $n_{x}=n_{y}-l$.

In the presence of both linear coupling and external non-linearities, in addition to the exchange of energy (transfer of instability growth rates between the transverse planes), there can also be a partition of Landau damping for "optimum" coupling. In Refs. 6 and 9, the influence of linear coupling on Landau damping of coherent instabilities has been assessed using two typical frequency distributions (Lorentzian, 
$\rho(f) \propto 1 /\left(1+u^{2}\right)$, and "elliptical", $\rho(f) \propto \sqrt{1-u^{2}}$ where $\left.u=\left(f-f_{0}\right) / \Delta f\right)$, knowing that they are limiting cases modeling spectra with and without important tails, and that realistic distributions are probably between them.

In the case of Lorentzian distributions, the necessary condition for the stability of the $m$ th mode and the stability criterion are given by Eqs. (8) and (9), replacing $V_{\text {eq } x, y}^{m}$ by $V_{\text {eq } x, y}^{m}-\delta \omega_{x, y}$, where $\delta \omega_{x, y}$ are the half widths at half maximum of the spectra.

In the case of elliptical distributions, the situation is more involved due to the finite tails. Two cases appear depending on whether the transverse coherent tunes (in the absence of coupling) are "far" from or "near" each other ("near" means a tune separation smaller than the order of magnitude of the average of the transverse spreads). If $Q_{h}$ is "far" from $Q_{v}+l$, then the necessary condition for the stability of the $m$ th mode and the stability criterion are given by Eqs. (8) and (9). There is no transfer of Landau damping since the coherent tunes are too far from each other to share their stabilizing spreads. If $Q_{h}$ is "near" $Q_{v}+l$, then in addition to the sharing of the instability growth rates, there is also a transfer of Landau damping for "optimum" coupling. The necessary condition for stability is

$$
\operatorname{Re}\left(\sqrt{\Delta \omega_{x}^{2}-\left(2 U_{\text {eqx }}^{m}\right)^{2}}+\sqrt{\Delta \omega_{y}^{2}-\left(2 U_{\text {eqy }}^{m}\right)^{2}}\right) \geq 2\left(V_{\text {eqx }}^{m}+V_{\text {eqy }}^{m}\right)
$$

where $\Delta \omega_{x, y}$ are the half widths at the bottom of the spectra. If Eq. (11) is true then it is possible to stabilize the beam and a condition similar to Eq. (9) for the stabilizing values of the coupling coefficient may be approximated by

$$
\begin{aligned}
& \left|\underline{\hat{K}}_{0}(l)\right| \approx \frac{\left(-Q_{x 0} Q_{y 0}\right)^{1 / 2}}{R^{2} \Omega_{0}} \times \\
& \left\{\begin{array}{l}
\left.\left[\operatorname{Re}\left(\sqrt{\Delta \omega_{x}^{2}-\left(2 U_{\text {equ }}^{m}\right)^{2}}\right)-2 V_{\text {eqx }}^{m}\right] \times\right\}^{1 / 2} \\
{\left[\operatorname{Re}\left(\sqrt{\Delta \omega_{y}^{2}-\left(2 U_{\text {eqy }}^{m}\right)^{2}}\right)-2 V_{\text {eqy }}^{m}\right]}
\end{array}\right\} .
\end{aligned}
$$

Notice that too strong coupling is detrimental here since it shifts the coherent tunes outside the spectra and thus prevents Landau damping.

Therefore, applying this theory, one sees from Figure 3 that the nominal singlebunch beam can be stabilized by linear coupling only (i.e. without octupoles), since for each mode, Eq. (8) is verified. Making the numerical computations, the stabilizing normalized skew gradient is given by $\left|\underline{\hat{K}}_{0}(0)\right|=\left|\underline{K}_{0}\right| \approx 10^{-5} \mathrm{~m}^{-2}$. Furthermore, one can notice that this result is still valid for "any" intensity (as concerns pure head-tail instability), since if the intensity is multiplied by a certain factor, the instability growth 
rates are both multiplied by the same factor and Eq. (8) remains then true. Notice also that this result is not modified by the transverse space-charge impedances (negative inductances), which have been neglected in this paper, since they do not affect the instability growth rates.

\section{Stabilization by Chromaticity Tuning}

Changes in machine chromaticity shift the beam oscillation spectrum centered at the chromatic frequency. The beam spectrum-impedance spectrum interaction is therefore modified and leads to different oscillation modes. It has been shown in Ref. 10, that a small gap in the horizontal chromaticity values $\left(\sim 0<\xi_{x}<\sim 0.05\right)$, where all modes are stable, should exist according to Sacherer's theory.

\section{Observations}

To insure the validity of Sacherer's one-dimensional theory, the skew quadrupole current must be set such as to have the minimum of linear coupling between the horizontal and vertical planes, i.e. $I_{\text {skew }} \approx 0.33 \mathrm{~A}$ [11]. Setting the octupole current to zero, a head-tail instability appeared with the single-bunch beam.

\section{Growth Rate Measurements and Determination of the Mode Number}

The instability was observed to be only in the horizontal plane. Figure 4(a) exhibits the first unstable betatron line, and Figure 4(b) shows that it is a head-tail instability with mode number $|m|=6$, which is in perfect agreement with theory.

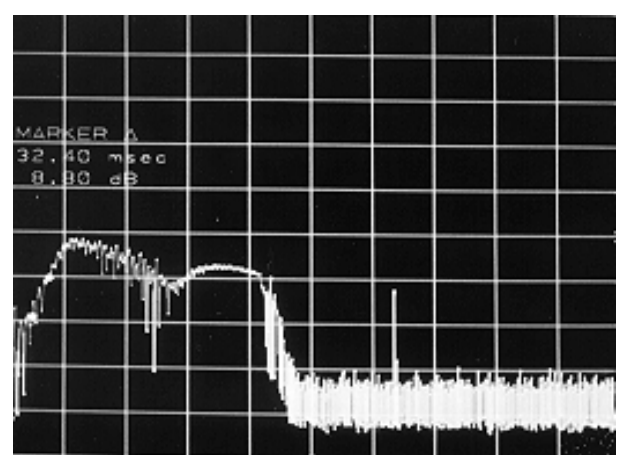

(a)

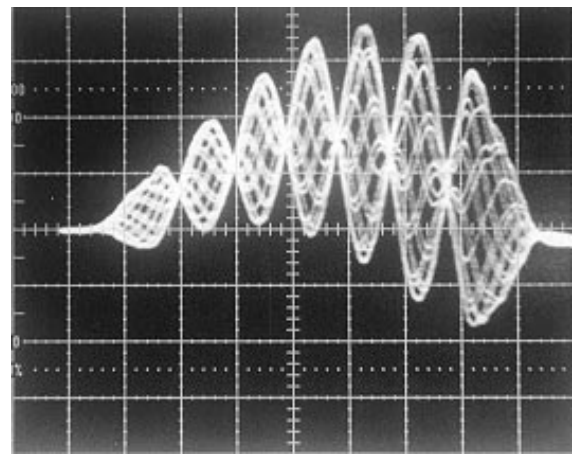

(b) 
FIGURE 4. (a) Measured horizontal instability growth rate on the first unstable betatron line (spectrum analyzer operating in zero frequency span) for the nominal single-bunch beam. Vertical scale: $10 \mathrm{~dB} /$ div. (b) $\Delta R$ signal from a radial beam-position monitor during 20 consecutive turns. Time scale: $20 \mathrm{~ns} / \mathrm{div}$.

\section{Stabilization by Landau Damping}

Tuning the octupole current, the instability could be damped. The results of the measurements compared to theory are collected in Table 2, which shows the measured and theoretical stabilizing octupole currents, and the ratio between the two.

TABLE 2. Measured and theoretical stabilizing octupole currents for the nominal singlebunch beam.

\begin{tabular}{ccc}
\hline$I_{o c t}{ }^{\exp }[\mathrm{A}]$ & $I_{o c t}{ }^{\text {theory }}[\mathrm{A}]$ & Ratio $=\left|\mathrm{I}_{o c t}{ }^{\text {exp }} / \mathrm{I}_{o c t}^{\text {theory }}\right|$ \\
\hline 8 & 6.6 & 1.2 \\
-10 & 6.6 & 1.5 \\
\hline
\end{tabular}

No emittance blow-up has been observed during the first $500 \mathrm{~ms}$, $\varepsilon_{x}^{\text {norm, } 1 \sigma} \approx \varepsilon_{y}^{\text {norm, } 1 \sigma} \approx 2 \mu \mathrm{m}$. The same analysis has been made for the ultimate singlebunch beam, i.e. with $N_{b} \approx 1.8 \times 10^{12}$ protons. The results of the stabilization by Landau damping are collected in Table 3. In this case also, no emittance blow-up has been observed during the first $500 \mathrm{~ms}, \varepsilon_{x}^{\text {norm, } 1 \sigma} \approx \varepsilon_{y}^{\text {norm, } 1 \sigma} \approx 3.2 \mu \mathrm{m}$.

TABLE 3. Measured and theoretical stabilizing octupole currents for the ultimate singlebunch beam.

\begin{tabular}{ccc}
\hline$I_{o c t}^{\exp }[\mathrm{A}]$ & $I_{o c t}{ }^{\text {theory }}[\mathrm{A}]$ & Ratio $=\mid \mathrm{I}_{o c t}{ }^{\text {exp }} / \mathrm{I}_{o c t}^{\text {theory }}$ \\
\hline 6 & 7.4 & 0.8 \\
-9 & 7.4 & 1.2 \\
\hline
\end{tabular}

\section{Stabilization by Linear Coupling}

By increasing the skew gradient instead of tuning the octupole current, the instability could also be damped, without emittance blow-up. The results of the measurements compared to theory are collected in Tables 4 and 5 for the nominal and ultimate beams. They both exhibit the measured stabilizing skew quadrupole current, its corresponding normalized skew gradient, the theoretical normalized skew gradient, and the ratio between the two.

TABLE 4. Measured and theoretical stabilizing normalized skew gradients for the nominal singlebunch beam.

\begin{tabular}{cccc}
\hline$I_{\text {skew }}[\mathrm{A}]$ & $\left|\underline{K}_{0}\right|^{\exp }\left(\times 10^{-5}\right)\left[\mathrm{m}^{-2}\right]$ & $\left|\underline{K}_{0}\right|^{\text {theory }}\left(\times 10^{-5}\right)\left[\mathrm{m}^{-2}\right]$ & Ratio $=\left|\underline{K}_{0}\right|^{\text {exp }} /\left|\underline{K}_{0}\right|^{\text {theory }}$ \\
\hline 0.73 & 1.7 & 1 & 1.7 \\
-0.07 & 1.7 & 1 & 1.7 \\
\hline
\end{tabular}

TABLE 5. Measured and theoretical stabilizing normalized skew gradients for the ultimate singlebunch beam.

$I_{\text {skew }}[\mathrm{A}] \quad \quad\left|\underline{K}_{0}\right|^{\text {exp }}\left(\times 10^{-5}\right)\left[\mathrm{m}^{-2}\right] \quad\left|\underline{K}_{0}\right|^{\text {theory }}\left(\times 10^{-5}\right)\left[\mathrm{m}^{-2}\right] \quad$ Ratio $=\left|\underline{K}_{0}\right|^{\text {exp }} /\left|\underline{K}_{0}\right|^{\text {theory }}$


\begin{tabular}{cccc}
0.68 & 1.5 & 1 & 1.5 \\
-0.02 & 1.5 & 1 & 1.5 \\
\hline
\end{tabular}

The relation between the skew quadrupole current and the modulus of the normalized skew gradient of the PS at $1 \mathrm{GeV}$ kinetic energy is given in Figure 5(a). For the present $1.4 \mathrm{GeV}$ kinetic energy, this measurement needs to be updated. However, a quick estimate has revealed that the minimum of linear coupling in the PS is obtained for the same skew quadrupole current, $I_{\text {skew }} \approx 0.33 \mathrm{~A}$. This result is in perfect agreement with those of Table 4 , where it can be seen that 0.73 and -0.07 are symmetric with respect to 0.33 , and thus correspond to the same skew gradient (as predicted by the stabilizing coupling theory). The future PS coupling measurement at $1.4 \mathrm{GeV}$ should reveal this feature. Anyhow, the new curve should not deviate from the one at $1 \mathrm{GeV}$ by more than $25 \%$, and Figure 5(a) can therefore be used in a first approximation. Furthermore, as the new energy is greater than the previous one, for the same level of skew quadrupole current, the normalized skew gradient should be smaller, which means that with the updated curve the agreement between theory and experiment should be even better.

$\left|\underline{K}_{0}\right|\left[\mathrm{m}^{-2}\right]$
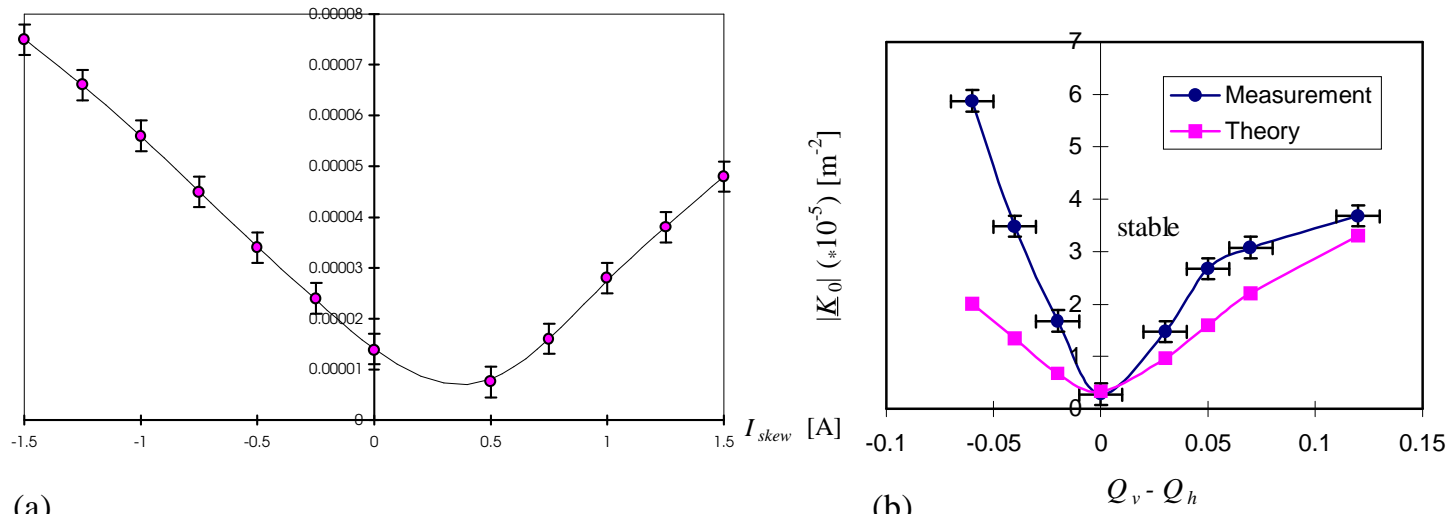

(a)

(b)

FIGURE 5. (a) Modulus of the normalized skew gradient vs. skew quadrupole current for the PS at $1 \mathrm{GeV}$ kinetic energy. (b) Stability boundary in the plane $\left|\underline{K}_{0}\right|$ vs. $Q_{v}-Q_{h}$ for the ultimate singlebunch beam.

As it can be seen from Eq. (9), the beam can be stabilized using the skew gradient and/or the tune separation. The results of damping measurements, made on the ultimate single-bunch beam, using both parameters are plotted in Figure 5(b).

\section{Chromaticity Tuning}

Using the pole-face-windings and figure-of-eight-loop in addition to the normal quadrupoles, the chromaticity could be changed. Figure 6 exhibits different unstable modes $(m=4,5,7,8,10)$ in the horizontal plane, in perfect agreement with Sacherer's theory, which have been obtained by tuning the chromaticity. However, one did not 
find stabilizing values of chromaticity, as could be predicted by the simplified Sacherer's theory.

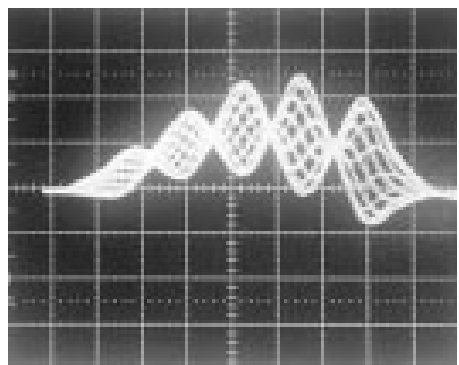

(a)

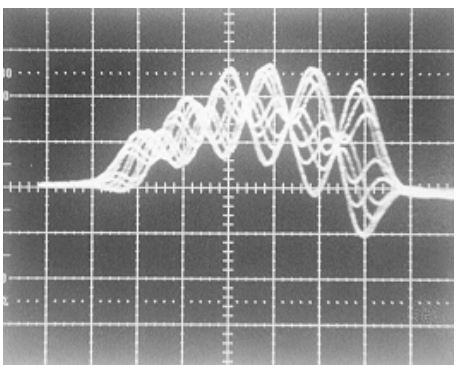

(b)

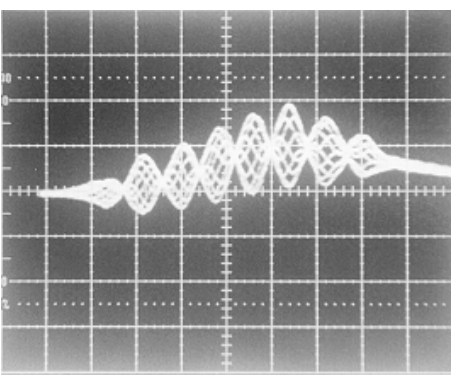

(c)

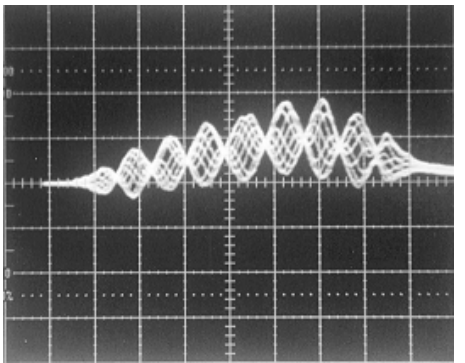

(d)

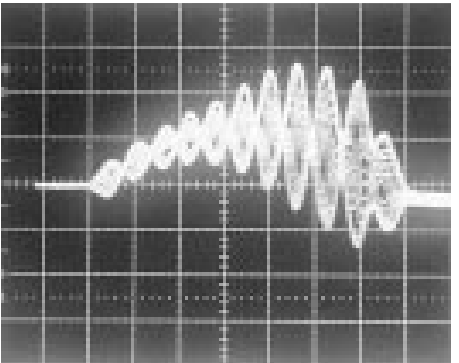

(e)

FIGURE 6. $\Delta R$ signal from a radial beam-position monitor during 20 consecutive turns. Time scale: $20 \mathrm{~ns} /$ div. (a) Nominal single-bunch beam with $Q_{h}=6.08, Q_{v}=6.32, \xi_{x} \approx-0.5$ and $\xi_{y} \approx-1.5$. (b) $Q_{h}=6.18, Q_{v}=6.21, \xi_{x} \approx-0.7$ and $\xi_{y} \approx-1.7$. (c) $Q_{h}=6.21, Q_{v}=6.18, \xi_{x} \approx-1.1$ and $\xi_{y} \approx-0.3$. (d) $Q_{h}=6.21, Q_{v}=6.16, \xi_{x} \approx-1.2$ and $\xi_{y} \approx 0.1$. (e) Ultimate single-bunch beam with $Q_{h}=6.20, Q_{v}=6.16, \xi_{x} \approx-1.3$ and $\xi_{y} \approx 0.1$.

\section{Future Predictions}

Applying Sacherer's formula, coupled-bunch instabilities should appear with the final beam, which will be composed of 8 bunches. The first unstable transverse betatron lines are such that $n_{x, y}=1$. The plot of the instability growth rates as functions of the head-tail mode number is represented in Figure 7 for the nominal beam. 


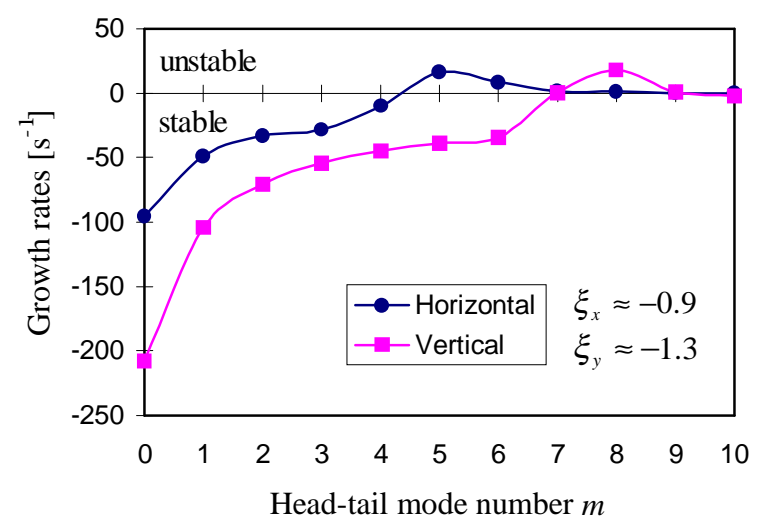

FIGURE 7. Transverse instability growth rates vs. head-tail mode number for the nominal 8 bunches beam.

One concludes therefore that the theory predicts transverse coupled-bunch instabilities $\left(n_{x, y}=1\right)$, with most critical head-tail mode number $m=5$ for the horizontal plane.

Using linear coupling, both modes $m=5$ and 6 should be stabilized, for $Q_{h}=6.18$ and $Q_{v}=6.21$, by putting $\left|\underline{K}_{0}\right| \approx 4.1 \times 10^{-5} \mathrm{~m}^{-2}$, i.e. tuning the skew quadrupole current to $I_{\text {skew }} \leq-0.68 \mathrm{~A}$ or $I_{\text {skew }} \geq 1.32 \mathrm{~A}$. However, under this condition, the modes $m=7,8$ and 9 should then become unstable, since both transverse instability growth rates are positive. One can perhaps imagine that these modes will be stabilized, remembering that Sacherer's theory is valid for the onset of coherent instabilities, and that the most critical modes will be damped by coupling. However, if a certain amount of octupole current is needed, it could be optimized using coupled Landau damping [12].

As concerns the final ( 8 bunches) ultimate beam, the transverse complex frequency shifts are multiplied by 1.8 (for the same coherent tunes), and the same results are obtained for the stabilization by linear coupling.

\section{Conclusions}

The stability criterion for the damping of transverse head-tail instabilities in the presence of linear coupling has been verified experimentally and compared to theory, leading to a good agreement (to within a factor smaller than 2).

The high-order head-tail instabilities of the CERN-PS beam for LHC (single bunch with nominal or ultimate intensity) can be damped using coupling only (skew quadrupoles and/or tune separation). Furthermore, this result is predicted by theory for "any" intensity (as concerns pure head-tail instability). 
The coupled-bunch instabilities should be damped also by coupling only (at least the most critical horizontal modes), or using coupled Landau damping (octupoles + coupling) to reduce the amount of external non-linearities.

\section{SPACE CHARGE}

Space charge tune shifts can convey the beam onto non-linear resonances generating transverse emittance blow-up. The e.g. horizontal incoherent tune shift of the particle located in the center of a (transversally) Gaussian bunch, is given by (neglecting the wall effects) [13]

$$
\Delta Q_{i n c, x 0}=-\frac{2 r_{p} I_{p} \beta_{x} R}{e c \beta^{3} \gamma^{3} a(a+b)} .
$$

A similar equation is obtained for the vertical plane, replacing $x$ by $y$ and reversing the roles of $a$ and $b$ in Eq. (13). Making the numerical computations, one obtains for the single-bunch beam with nominal intensity, $\Delta Q_{i n c, x 0}=-0.18$ and $\Delta Q_{i n c, y 0}=-0.21$. For the single-bunch beam with ultimate intensity, it yields $\Delta Q_{i n c, x 0}=-0.22$ and $\Delta Q_{i n c, y 0}=-0.25$. The modulus of the previous values are below 0.3 , and therefore the absence of blow-up due to resonance crossings is in agreement with what was expected in Ref. 13.

As concerns head-tail instabilities, it has been shown before that the onedimensional (horizontal) theory of Landau damping is in agreement with the observations if the space-charge impedance, given by $Z_{x}^{S C}(\omega)=-j R Z_{0}\left(a_{\text {round }}^{-2}-b_{\text {round }}^{-2}\right) / \beta^{2} \gamma^{2}$ for the simplified case of a round beam of radius $a_{\text {round }}$ circulating in a round pipe of radius $b_{\text {round }}$, is neglected (or at least the first incoherent term). Further work is needed to investigate this feature.

During the experiments, it has also been verified that the spread of the incoherent tune shift alone has no stabilizing effect on the high-order head-tail instabilities, as expected [7]. For the nearly round single-bunch beam with nominal intensity, a simple estimate for the e.g. horizontal space-charge tune spread, is given in Ref. 7, considering elliptical cross-section and parabolic density,

$$
\Delta Q_{x}^{\text {sc spread }} \approx-\frac{\sqrt{13}}{8} \Delta Q_{i n c, x 0}
$$

From the numerical computations, $\Delta Q_{x}^{\text {scspread }} \approx 0.08$, which should largely damp the head-tail instability $m=6$, if the criterion of Eq. (6) could be used with the internal spread only. In practice, the instability is not damped in the absence of both octupoles and linear coupling, which shows that external non-linearities are required for Landau damping. 


\section{CONCLUSION}

Theoretical and experimental studies have been made on the longitudinal and transverse stability problems in the CERN-PS beam for the LHC. The longitudinal coupled-bunch instability can be damped by a longitudinal feedback. The longitudinal microwave instability will be avoided by adopting a new scheme, which is under study, to produce the LHC bunch train.

As concerns the transverse domain, until now experiments have been performed on a single-bunch beam with nominal and ultimate intensities. In both cases, linear coupling is sufficient to damp the high-order head-tail instabilities (in agreement with theory), without emittance blow-up. The next step consists in studying the final eight bunches beam.

\section{ACKNOWLEDGEMENTS}

We wish to thank S. Hancock, G. Métral and R. Steerenberg for their help during the experiments.

\section{REFERENCES}

1. K. Schindl, "The Injector Chain for the LHC", CERN/PS 99-018 (DI), 1999.

2. A. Blas et al., "Beams in the CERN PS Complex after the RF Upgrades for LHC", CERN/PS 98022 (RF) or $6^{\text {th }}$ European Particle Conference (EPAC98), June $22-26$, 1998, Stockholm, Sweden.

3. F. Pedersen and F. Sacherer, "Theory and Performance of the Longitudinal Active Damping System for the CERN PS Booster", CERN-PS-BR-77-8, 1977.

4. R. Garoby, "Bunch Merging and Splitting Techniques in the Injectors for High Energy Hadron Colliders", CERN/PS 98-048 (RF) or $17^{\text {th }}$ International Conference on High Energy Accelerators (HEACC'98), September 7-12, 1998, Dubna, Russia.

5. F. Sacherer, "Transverse Bunched-Beam Instability-Theory", Proc. $9^{\text {th }}$ Int. Conf. on High Energy Accelerators, Stanford 1974 (CONF 740522, US Atomic Energy Commission, Washington D.C., 1974), p. 347.

6. E. Métral, "Coupled Landau Damping of Transverse Coherent Instabilities in Particle Accelerators", Thesis, Joseph Fourier University of Grenoble (France), 1999.

7. D. Möhl, "On Landau Damping of Dipole Modes by Non-Linear Space Charge and Octupoles", CERN/PS 95-08 (DI), 1995.

8. A.W. Chao, Physics of Collective Beam Instabilities in High Energy Accelerators, New York: Wiley, 371 p, 1993.

9. E. Métral, "Theory of Coupled Landau Damping”, Part. Accelerators, 62(3-4), p. 259, 1999. 
10. R. Cappi, "Observations of High-Order Head-Tail Instabilities at the CERN-PS", CERN/PS 95-02 (PA), 1995.

11. E. Métral, "Measurement of the PS Linear Coupling using FFT Analysis", CERN/PS/CA/Note 9724, 1997.

12. E. Métral, "Measurements of Coupled Landau Damping of the PS Coherent Resistive Instability", CERN/PS/CA/Note 98-16, 1998.

13. R. Cappi, “The PS in the LHC Injector Chain”, CERN/PS 97-16 (CA), 1997.

\section{APPENDIX: List of PS and beam parameters during the experiments}

$$
\begin{aligned}
& a=\sqrt{2}\left[\varepsilon_{x}^{\mathrm{rms}} \beta_{x}+\left(D_{x} \sigma_{p} / p\right)^{2}\right]^{1 / 2} \\
& a_{w}=7 \mathrm{~cm} \\
& b=\sqrt{2} \sqrt{\varepsilon_{y}^{\text {rms }} \beta_{y}} \\
& b_{w}=3.5 \mathrm{~cm} \\
& c=3 \times 10^{8} \mathrm{~ms}^{-1} \\
& D_{x} \approx 2.5 \mathrm{~m} \\
& D_{y} \approx 0 \mathrm{~m} \\
& e=1.6 \times 10^{-19} \mathrm{C} \\
& E_{0}=0.938 \mathrm{GeV} \\
& E_{c}=1.4 \mathrm{GeV} \\
& I_{b} \\
& I_{p}=3 e N_{b} / 2 \tau_{b} \\
& j=\sqrt{-1} \\
& k
\end{aligned}
$$

$$
\begin{aligned}
& L \\
& m=\ldots,-1,0,1, \ldots \\
& m_{0}=1.67 \times 10^{-27} \mathrm{~kg} \\
& M \\
& n_{x, y}=0,1, \ldots, M-1 \\
& N_{b} \\
& N_{\text {oct }}=8 \\
& Q \approx 1 \\
& Q_{h}=6.18 \\
& Q_{v}=6.21 \\
& Q_{x 0, y 0} \\
& r_{p}=1.54 \times 10^{-18} \mathrm{~m} \\
& R=100 \mathrm{~m} \\
& R_{x} \approx 1 \mathrm{M} \Omega / \mathrm{m}
\end{aligned}
$$

$\sqrt{2} \times$ the rms horizontal beam dimension

Half major axis of the elliptical beam pipe

$\sqrt{2} \times$ the rms vertical beam dimension

Half minor axis of the elliptical beam pipe

Velocity of light

Average horizontal dispersion function

Average vertical dispersion function

Elementary charge

Proton rest energy

Proton kinetic energy

Current in one bunch

Bunch peak current considering a parabolic line density

Imaginary unit

$k=\ldots,-1,0,1, \ldots$ for a single bunch or several bunches oscillating independently; $k=n_{x, y}+k^{\prime} M \quad$ with $k^{\prime}=\ldots,-1,0,1, \ldots$ for coupled motion of $M$ bunches

Bunch length (in meters)

Head-tail mode number

Proton rest mass

Number of bunches of the beam

Transverse coupled-bunch mode numbers

Bunch intensity. $N_{b} \approx 10^{12}$ protons for the nominal beam; $N_{b} \approx 1.8 \times 10^{12}$ protons for the ultimate beam

Number of octupoles

Quality factor of the broadband impedances

Horizontal coherent tune

Vertical coherent tune

Transverse coherent tunes in the absence of wake fields

Classical proton radius

Average radius of the machine

Shunt resistance of the horizontal broadband impedance 


$$
\begin{aligned}
& R_{y} \approx 3 \mathrm{M} \Omega / \mathrm{m} \\
& Z_{0}=377 \Omega \\
& \alpha_{p}=\gamma_{t r}^{-2}=0.027 \\
& \beta=0.916 \\
& \beta_{\text {octx }} \approx 12.4 \mathrm{~m} \\
& \beta_{\text {octy }} \approx 22.6 \mathrm{~m} \\
& \beta_{x} \approx R / Q_{h}=16.2 \\
& \beta_{y} \approx \mathrm{R} / \mathrm{Q}_{v}=16.1 \\
& \gamma=2.493 \\
& \varepsilon_{0}=8.84 \times 10^{-12} \mathrm{Fm}^{-1} \\
& \varepsilon_{x, y}^{\text {norm, } 1 \sigma}=\beta \gamma \varepsilon_{x, y}^{\mathrm{ms}} \\
& \varepsilon_{x, y}^{\mathrm{mms}}
\end{aligned}
$$$$
\eta=\alpha_{p}-\gamma^{-2}=-0.134
$$$$
\xi_{x} \approx-0.9
$$$$
\xi_{y} \approx-1.3
$$$$
\rho_{w}=9 \times 10^{-7} \Omega \mathrm{m}
$$$$
\tau_{b}=160 \mathrm{~ns}
$$$$
\omega_{r}=2 \pi f_{r}=2 \pi \times 1.4 \mathrm{GHz}
$$$$
\omega_{s}=2 \pi f_{s}=2 \pi \times 610 \mathrm{~Hz}
$$$$
\omega_{\xi_{x, y}}=\left(\xi_{x, y} / \eta\right) Q_{x 0, y 0} \Omega_{0}
$$$$
\Omega_{0}=2 \pi f_{0}=2 \pi \times 436.5 \mathrm{kHz}
$$$$
B_{v} \rho_{x}=7.14 \mathrm{Tm}
$$$$
\sigma_{p} / p \approx 10^{-3}
$$$$
\operatorname{Sgn}(\omega)=1 \text { if } \omega>0,-1 \text { if } \omega<0
$$$$
\int_{0}^{l_{\operatorname{mot}}} K_{o c t} d l\left[\mathrm{~m}^{-3}\right]=\frac{4.33}{6 B_{y} \rho_{x}} \times I_{o c t}[\mathrm{~A}]
$$

Shunt resistance of the vertical broadband impedance

Free space impedance

Momentum compaction factor

Relativistic velocity factor

Horizontal betatron function at the octupoles

Vertical betatron function at the octupoles

Average horizontal betatron function

Average vertical betatron function

Relativistic mass factor

Permittivity of free space

Normalized rms transverse emittances

Rms transverse emittances. $\varepsilon_{x}^{\mathrm{ms}}=0.83 \times 10^{-6} \mathrm{~m}$ and $\varepsilon_{y}^{\mathrm{rms}}=0.87 \times 10^{-6} \mathrm{~m} \quad$ for the nominal beam; $\varepsilon_{x}^{\mathrm{ms}}=1.36 \times 10^{-6} \mathrm{~m} \quad$ and $\quad \varepsilon_{y}^{\mathrm{rms}}=1.40 \times 10^{-6} \mathrm{~m}$ for the ultimate beam

Slippage (or off-momentum) factor

Horizontal (relative) chromaticity

Vertical (relative) chromaticity

Vacuum chamber resistivity

Total bunch length (in seconds)

Vacuum chamber cut-off (angular) frequency

Synchrotron (angular) frequency of the particles

Transverse chromatic (angular) frequencies

Average revolution (angular) frequency of the particles

Beam rigidity

Rms relative momentum spread

Integrated octupole strength 